\title{
Heterogeneous beliefs, wealth accumulation, and asset price dynamics
}

\author{
Antonio Cabrales, a , Takeo Hoshi ${ }^{\text {b }}$ \\ a'Department d'Economia, Universitat Pompeu Fabra, 08008 Barcelona, Spain \\ ${ }^{\circ}$ Graduate School of International Relations and Pacific Studies, University of California, San Diego, \\ CA 92093, USA
}

(Received March 1994; final version received September 1995)

\begin{abstract}
This paper develops and analyzes a model of asset markets with two types of investors. We study the stochastic processes for the distribution of wealth between the two types of investors and for the equilibrium asset returns. The relationship between this model and some econometric models with time varying parameters, such as the ARCH (Autoregressive Conditional Heteroskedasticity) model, as well as the relationship between the volume of trade and volatility, are examined. The dynamic properties of another model, regarding investors who use strategies that are a bit more complex, are also analyzed.
\end{abstract}

Key words: Asset pricing; Dynamics; Heterogeneity; ARCH; Trade volume JEL classification: $\mathrm{G} 10 ; \mathrm{G} 12$

\section{Introduction}

Recent studies in finance, which include Shiller (1984), Black (1986), Frankel and Froot (1988, 1990a, b), and De Iong, Shleifer, Summers, and Waldmann

*Corresponding author.

The authors would like to thank Valentina Corradi, Dean Foster, Daniel Friedman, Alex Kane, Bruce Lehman, Albert Marcet, Makoto Saito, Joel Sobel, and seminar audiences at University of California, San Dicgo, Tohoku University, Brown University, Universitat Pompeu Fabra, and Universidad Complutense de Madrid for their helpful comments. Hoshi acknowledges the financial support from the UCSD Academic Senate. Cabrales acknowledges the financial support of the DGICYT under grant PB93-0398. 
(DSSW henceforth) (1990) among others, stressed the importance of heterogeneous beliefs in asset markets. These studies show that models with heterogeneous investors are useful in explaining some empirical puzzles that cannot be explained by a model with a representative investor.

This paper studies a similar model with heterogeneous beliefs to analyze the asset price dynamics. We consider an asset market which includes two types of investors, who follow two different rule of thumb strategies. Depending on the relative success of each strategy, the proportion of total wealth held by each type of investor fluctuates. If strategy A is more successful than the other strategy B, for example, the investors who use strategy $\mathrm{A}$ increase their wealth more than the other investors, invest more in the asset, and increase their influence on asset pricing. Thus, the changes in the distribution of wcalth between the two types of investors affect the asset prices, which in turn influence the relative success of each strategy and again change wealth distribution. Profits, which are random, are used for buying back the shares of the risky asset to create capital gains for the owners, or for paying out dividends to create income gains. Thus, random profit shocks influence the dynamics of the asset price and wealth distribution. The asset price and wealth distribution themselves also become stochastic processes. This paper studies the limiting behavior of these stochastic processes. Under some parameter values, we show that the limiting behavior of those dynamics does not depend on the initial condition.

The most important empirical implication of our model is smooth shifts of the price dynamics. Both the conditional mean and the conditional variance of the asset price change over time reflecting changes in the wealth distribution between two types of investors. For some periods, strategy A may do better than strategy $B$ and enhance its influence. Following some random shocks to profits, strategy B may become more profitable, and the proportion of wealth held by investors who use strategy B will increase. Following some other shocks, strategy A may again become more profitable and regain its influence. In this way, wealth distribution fluctuates over time and asset price dynamics show smooth shifts between two extreme regimes: one where the wealth owned by investors who use strategy $A$ is infinitely larger than the wealth owned by investors who use strategy $B$, and the other where the wealth owned by investors who use strategy $B$ is infinitely larger than the wealth owned by investors who use strategy A. If the limiting distribution of the proportion of wealth is ergodic, the price dynamics can show substantial variation over time without reaching an extreme. It has to be noted that even if the distribution of the proportions of wealth converges to one of the extremes, the wealth of the individuals does not go to zero with positive probability, it is only relative wealth that might go to zero under some parameter configurations.

Our model is useful in explaining an apparent empirical regularity in financial markets: namely time-varying conditional variance of asset returns. Timevarying conditional variance has been detected by many researchers who 
estimated ARCH (Autoregressive Conditional Heteroskedasticity) models to financial data. Bollerslev, Chou, and Kroner (1992) surveyed numerous successful applications of ARCH models in finance. The model with the representative agent does not usually have strong implications for the dynamics of conditional variance of asset returns. Thus, in order to interpret empirical success of ARCH models in finance, the standard model has to assume ARCH at the fundamental level, for example in the process of cash flow. The model in this paper can explain time-varying conditional variance without assuming $\mathrm{ARCH}$ at the fundamental level. The model also implies that the mean growth rate of asset price changes over time, and changes in the mean are related to changes in the conditional variance. Thus, a type of ARCH models called the ARCH-M model, which is used to capture shifts in the conditional mean associated with changes in the conditional variance, becomes especially relevant for our model.

Our model is also useful in motivating another statistical model with timevarying parameters called STAR (Smooth Transition Autoregressive) model. The STAR model was applied to some aggregate variables like GNP and industrial production by Anderson and Teräsvirta (1991). A STAR model assumes that a process is a weighted average of the two distinct AR processes and the weight changes over time. Our model also implies that the asset price process shifts between two extremes, although the variable that determines the weight in our model (proportion of wealth) enters the price process in a complex way.

Another empirical implication of the model is on the relationship between volume of trade and asset return volatility. We show that it is possible, under certain parameter values, for the conditional mean and variance of the volume of trade to be positively correlated with volatility, as Gallant, Rossi, and Tauchen (1992), among others, find.

There are several studies of the dynamics of asset prices in the asset market with heterogeneous agents. DSSW (1991) considers the wealth accumulation by two types of investors, noise traders, and sophisticated investors. The noise traders have random and biased forecasts of the rate of return and its variance, whereas the sophisticated investors have the correct forecasts. They show, under some parameter values, that the noise traders' wealth may grow faster than that of the sophisticated investors and eventually the noise traders may dominate the market. One problem of DSSW (1991) is that they ignore the noise traders' influence on the asset price, which is the most important point made by models with noise traders, such as DSSW (1990). Our model explicitly studies the way the heterogeneous beliefs influence asset prices and examines the price dynamics.

Another important difference between our paper and DSSW's research is that we do not assume the presence of sophisticated investors who have rational expectations and maximize their expected utility. Although the agents in our model use portfolio strategies that maximize an expected utility under some assumptions about beliefs, they do not have rational expectations. Thus, the 
agents in this paper deviate from the standard rational consumers in economics. In this sense, our approach is similar to that followed by Blume and Easley (1992). They consider the dynamic process of an asset market with heterogeneous investors, which is similar to ours. Each type of investor uses different portfolio rules, and the market eventually selects the most 'fit' rules, in the sense that wealth held by such investors grows faster than that of the other investors. One difference between our model and Blume and Easley (1992) is the formulation of asset return. Blume and Easley (1992) assume an exogenous probability distribution over the possible pay-out of the asset. Thus, the current price of the asset does not influence the rate of return on an asset that was bought last period. In other words, the assets in their model can have only income gains and not capital gains. Our model includes both income gains and capital gains of asset holding. Although we assume nonrationality of agents, the assumption is taken for its analytical convenience. Our results on the dynamics of asset prices and the survival of heterogeneous agents in the market do not depend on agents being nonrational; the important assumption is that they are heterogeneous. To show that this is indeed the case, we provide an example where agents have heterogeneous preferences in the Appendix. The results are similar to others obtained in the paper.

The paper is organized as follows. The next section presents a model of an asset market with two types of investors: optimists who expect a high rate of return for the risky asset and pessimists who expect a low rate of return for the risky asset. The dynamics of the asset price and wealth distribution are derived and characterized. Section 3 studies the asymptotic distribution of the proportion of wealth held by each agent. Section 4 discusses the implications on conditional variance and relates our model to ARCH models in econometrics. Section 5 examines the relationship between trade volume and volatility implied by the model. Section 6 considers the investors who use slightly more complex strategies. 'Fundamentalists' behave similarly to optimists and pessimists in Section 2. They maximize the expected utility given their beliefs about the process of the asset returns. 'Contrarians' start with the assumption that the market on average is wrong, and invest a proportion of their wealth in the risky asset which is opposite to the average proportion of wealth invested in that asset. The price dynamics in a market with fundamentalists and contrarians is examined. Section 7 contains some concluding remarks. The Appendix contains the example of a model with rational agents who have heterogeneous preferences and the proof of the propositions.

\section{Optimists, pessimists, and the asset price}

We consider an asset market where there are two types of investors. One type of investor, called 'optimist', holds higher expectation about the rate of return of 
the risky asset than the other type of investor, called 'pessimist'. Besides this assumption of heterogeneous beliefs, our model is very much a standard model of asset pricing used in macro/finance literature. ${ }^{1}$ Time is continuous and investors have infinite horizons. Both types of agents are assumed to have identical preferences with constant relative risk aversion, so their instantaneous utility function is $u(C)=C^{1-\tau} /(1-\tau), \tau \neq 1$, and $u(C)=\log C$ for $\tau=1$, where $\tau(\geqslant 0)$ is the coefficient of relative risk aversion and $C$ denotes consumption. There are two types of assets, a riskless asset with an instantaneous rate of return $r$ and a risky asset whose price is $P(t)$. We assume that the riskless asset has unlimited supply at $r$, and the risky asset is the claim to the single firm operating in this economy. Let $\mathrm{d} D(t)$ be instantaneous dividends per share. Let $W^{\circ}(t)$ be the wealth of the optimists, $W^{r}(t)$ the wealth of the pessimists, and $W(t)=W^{\mathrm{o}}(t)+W^{\mathfrak{p}}(t)$. Let $\lambda^{i}(t)$ be the proportion of wealth that an agent of type $i$ invests in the risky asset and $c^{i}(t)$ the proportion consumed out of total wealth per unit of time. Given the assumptions about the return of the assets, wealth evolves according to the following equation:

$$
\begin{array}{r}
\mathrm{d} W^{i}(t)=\left(\lambda^{i}(t) \frac{\mathrm{d} P(t)+\mathrm{d} D(t)}{P(t)}+\left(1-\lambda^{i}(t)\right) r \mathrm{~d} t-c^{i}(t) \mathrm{d} t\right) \\
W^{i}(t), \\
i=0, \mathrm{p} .
\end{array}
$$

The agents believe that the returns of the risky asset follow the stochastic process given by

$$
\frac{\mathrm{d} P(t)+\mathrm{d} D(t)}{P(t)}=\alpha_{i} \mathrm{~d} t+\beta \mathrm{d} B(t)
$$

where $i$ is equal to $o$ for the optimists and $\mathrm{p}$ for the pessimists and $\alpha_{\mathrm{o}}>\alpha_{\mathrm{p}}$. Thus, optimists expect higher returns than pessimists for a given level of risk. We assume that the agents never change their beliefs. The influence of each belief on the asset price, however, changes as the wealth distribution between two types of agent changes. It may be more realistic to assume that some agents change their beliefs if their strategy yields much lower returns than the alternative one, but such an assumption would not change the qualitative results. Under such an assumption, a successful strategy would increase the influence through two channels. The wealth of the agents that use the strategy grows faster, and, at the

\footnotetext{
${ }^{1}$ The crucial assumption for our purpose is heterogeneity in general, not necessarily in beliefs. The Appendix presents an example where agents are symmetrically informed and have rational expectations, but have heterogeneous risk preferences. The conclusions from such a model are qualitatively the same as those obtained from the model in the text.
} 
same time, the strategy gains some new converts. Thus, the dynamics of the model would look qualitatively the same.

The objective of the agents is to maximize $\mathrm{E}\left\{\int_{0}^{\infty} \mathrm{e}^{-\rho s} u[C(s)] \mathrm{d} s\right\}$ subject to the budget constraint (1), and $C^{i}(t)>0, W^{i}(t)>0, W^{i}(0)=W_{0}>0$. Under these conditions and an additional assumption $\rho>(1-\tau)\left[\left(\left(\alpha_{i}-r\right)^{2} / 2 \sigma^{2} \tau\right)+r\right]$ (which always holds when the agent is more risk-averse than an agent with logarithmic utility) the consumption by each type of agent will be a constant proportion of their total wealth (see Merton, 1969):

$$
c^{i}=\frac{1}{\tau}\left(\rho-(1-\tau)\left[\left(\left(\alpha_{i}-r\right)^{2} / 2 \sigma^{2} \tau\right)+r\right]\right)
$$

Merton (1969) also shows that the proportion of the risky asset in the portfolio of a type $i$ agent, $\lambda^{i}$, is given by $\left(\alpha_{i}-r\right) / \tau \beta^{2}$.

Let $N(t)$ be the total number of shares outstanding at time $t$. Then, market clearing implies

$$
\lambda^{\circ} W^{\circ}(t)+\lambda^{\mathrm{p}} W^{\mathrm{p}}(t)=P(t) N(t)
$$

or if we let $q(t)$ denote the proportion of wealth held by the optimists, i.e., $W^{\circ}(t) / W(t)$.

$$
\lambda^{0} q(t)+\lambda^{\mathrm{p}}(1-q(t))=N(t) P(t) / W(t) .
$$

The firm's instantaneous profits, $\mathrm{d} \pi^{*}(t)$, are assumed to follow the following stochastic process:

$$
\mathrm{d} \pi^{*}(t)=\pi^{\mathrm{o}} W^{\mathrm{o}}(t) \mathrm{d} t+\pi^{\mathrm{p}} W^{\mathrm{p}}(t) \mathrm{d} t+\sigma W(t) \mathrm{d} B(t),
$$

which, by the definition of $q(t)$, can be written as

$$
\begin{aligned}
\mathrm{d} \pi^{*}(t) & =\left(\left(\pi^{\mathrm{o}} q(t)+\pi^{\mathrm{p}}(1-q(t))\right) \mathrm{d} t+\sigma \mathrm{d} B(t)\right) W(t) \\
& =(\pi(q(t)) \mathrm{d} t+\sigma \mathrm{d} B(t)) W(t),
\end{aligned}
$$

where $\pi^{\mathbf{o}}, \pi^{\mathrm{p}}$, and $\sigma$ are constant parameters. ${ }^{2}$ The firm uses the profits to buy back its shares and/or pay out dividends, so that

$$
\mathrm{d} \pi^{*}(t)=-\mathrm{d} N(t)(P(t)+\mathrm{d} P(t))+N(t) \mathrm{d} D(t) .
$$

\footnotetext{
${ }^{2}$ To motivate this assumption, suppose for the moment that the firm is a monopolist in a contestable market, so that it charges the price just low enough to deter new entrants. This assumption, combined with an implication of the constant relative risk aversion utility function that says the consumers spend a constant proportion of wealth for consumption goods, implies profits are also
} 
Using the market equilibrium condition (2) and the relation between asset returns and the profits process given by (4), we can solve for the process of equilibrium asset returns. Then, by using (1) and Itô's formula, we can derive the stochastic process for $q(t)$, the proportion of wealth held by the optimists. ${ }^{3}$

Proposition 1.

a)

$$
\begin{aligned}
\frac{\mathrm{d} P(t)+\mathrm{d} D(t)}{P(t)}= & \frac{\pi(q(t))-\lambda^{0} c^{o} q(t)-\lambda^{\mathrm{p}} c^{\mathrm{p}}(1-q(t))}{\lambda^{\mathrm{o}}\left(1-\lambda^{\mathrm{o}}\right) q(t)+\lambda^{\mathrm{p}}\left(1-\lambda^{\mathrm{p}}\right)(1-q(t))} \mathrm{d} t+r \mathrm{~d} t \\
& +\frac{1}{\lambda^{\mathrm{o}}\left(1-\lambda^{\mathrm{o}}\right) q(t)+\lambda^{\mathrm{p}}\left(1-\lambda^{\mathrm{p}}\right)(1-q(t))} \sigma \mathrm{d} B(t)
\end{aligned}
$$

b) $\quad \mathrm{d} q(t)=q(t)(1-q(t))\left(\lambda^{\mathrm{o}}-\lambda^{\mathrm{p}}\right)\left[\frac{\pi(q(t))-\lambda^{\mathrm{o}} c^{\mathrm{o}} q(t)-\lambda^{\mathrm{p}} c^{\mathrm{p}}(1-q(t))}{\lambda^{\mathrm{o}}\left(1-\lambda^{\mathrm{o}}\right) q(t)+\lambda^{\mathrm{p}}\left(1-\lambda^{\mathrm{p}}\right)(1-q(t))}\right.$

$$
\begin{aligned}
& \left.-\frac{c^{\mathrm{o}}-c^{\mathrm{p}}}{\lambda^{\mathrm{o}}-\lambda^{\mathrm{p}}}\right] \mathrm{d} t-\frac{q(t)(1-q(t))\left(\lambda^{\mathrm{o}}-\lambda^{\mathrm{p}}\right)\left(\lambda^{\mathrm{o}} q(t)+\lambda^{\mathrm{p}}(1-q(t)) \sigma^{2}\right.}{\left(\lambda^{\mathrm{o}}\left(1-\lambda^{\mathrm{o}}\right) q(t)+\lambda^{\mathrm{p}}\left(1-\lambda^{\mathrm{p}}\right)(1-q(t))\right)^{2}} \mathrm{~d} t \\
& +\frac{q(t)(1-q(t))\left(\lambda^{\mathrm{o}}-\lambda^{\mathrm{p}}\right) \sigma}{\lambda^{\mathrm{o}}\left(1-\lambda^{\mathrm{o}}\right) q(t)+\lambda^{\mathrm{p}}\left(1-\lambda^{\mathrm{p}}\right)(1-q(t))} \mathrm{d} B(t),
\end{aligned}
$$

Proof. See the Appendix.

The proposition shows that the equilibrium asset returns depend on random shocks to the profits. More importantly, the proportion of optimists' wealth, $q(t)$, affects the equilibrium asset returns. The dynamics of $q(t)$ is given by the latter half of the proposition.

proportional to wealth. We add the noise term to incorporate other factors not taken into account by the ones included in the drift term. We assume the variance of the noise depends on wealth. Examples of such noise would include the effect of scientific discoveries on costs and some government intervention on the business, which are likely to be related to the total wealth in the economy. Linearity and additivity are assumed for analytical tractability.

${ }^{3}$ Itô's rule is the analog in stochastic differential calculus to the chain rule in ordinary calculus. Let $X(t)$ be a stochastic integral represented by $\mathrm{d} X(t)=u \mathrm{~d} t+v \mathrm{~d} B(t)$ in its stochastic differential form.

Let $g(t, x)$ be a twice continuously differentiable function. Then, $Y(t)=g(t, X(t))$ is also a stochastic integral, and

$$
\mathrm{d} Y(t)=\frac{\partial g}{\partial t}(t, X(t)) \mathrm{d} t+\frac{\partial g}{\partial x}(t, X(t)) \mathrm{d} X(t)+\frac{1}{2} \frac{\partial^{2} g}{\partial x^{2}}(t, X(t))(\mathrm{d} X(t))^{2},
$$

where $\mathrm{d} t \cdot \mathrm{d} t=\mathrm{d} t \cdot \mathrm{d} B(t)=\mathrm{d} B(t) \cdot \mathrm{d} t=0, \mathrm{~d} B(t) \mathrm{d} B(t)=\mathrm{d} t$. See, for example, Øksendal (1989). 
The next section studies the asymptotic properties of the dynamics of $q(t)$. Since the dynamics of asset returns critically depends on that of $q(t)$, the knowledge of asymptotic properties of $q(t)$ is necessary for us to understand the long-run properties of asset return dynamics. To simplify the notation, we hereafter write the dynamics of $q(t)$, given by the latter equation of the proposition, as

$$
\mathrm{d} q(t)=a(q(t)) \mathrm{d} t+b(q(t)) \mathrm{d} B(t)
$$

\section{Asymptotic behavior of the model}

This section investigates asymptotic properties of the process $q(t)$. Let us first define the following expressions which are useful in examining the asymptotic properties:

$$
\begin{aligned}
& I_{1}=\int_{0}^{q(0)} \exp \left[-\int_{z}^{x} \frac{2 a(q)}{b(q)^{2}} \mathrm{~d} q\right] \mathrm{d} x \\
& I_{2}=\int_{q(0)}^{1} \exp \left[-\int_{z}^{x} \frac{2 a(q)}{b(q)^{2}} \mathrm{~d} q\right] \mathrm{d} x \\
& M(x)=\frac{2}{b(x)^{2}} \exp \left[\int_{z}^{x} \frac{2 a(q)}{b(q)^{2}} \mathrm{~d} q\right]
\end{aligned}
$$

Here $z$ is an arbitrary number in $(0,1)$. As Gihman and Skorohod (1972) show, $I_{1}, I_{2}, \int_{0}^{1} M(x) \mathrm{d} x$, and the asymptotic properties of the process $q(t)$ have the following relations. ${ }^{4}$ (1) If $I_{1}$ is infinite and $I_{2}$ is finite, $q(t)$ converges to one almost surely. (2) If $I_{1}$ is finite and $I_{2}$ is infinite, $q(t)$ converges to zero almost surely. (3) If $I_{1}$ and $I_{2}$ are finite, $q(t)$ converges to one with probability $I_{1} /\left(I_{1}+I_{2}\right)$ and to zero with probability $I_{2} /\left(I_{1}+I_{2}\right)$. (4) If $I_{1}$ and $I_{2}$ are infinite, the process converges to neither zero nor one; if in addition $\int_{0}^{1} M(x) \mathrm{d} x$ is finite, $q(t)$ has a unique ergodic distribution with density $M(x) / \int_{0}^{1} M(y) \mathrm{d} y$. When both $I_{1}$ and $I_{2}$ are infinite but $\int_{0}^{1} M(x) \mathrm{d} x$ is also infinite, there will be an ergodic distribution but it will not be unique, it will be depend on the value of $q(0)$.

The relation between these measures and the asymptotic properties of $q(t)$ can be intuitively understood. The function $I_{1}$ measures the difficulty for $q(t)$ to

\footnotetext{
${ }^{4}$ This approach was used by Fudenberg and Harris (1992) to study the asymptotic properties of a class of evolutionary game dynamics.
} 
converge to zero. A large $I_{1}$ means that $a(q) / b(q)^{2}$ is positive when $q$ is close to zero. Thus, the positive deterministic drift, captured by $a(q)$, tends to dominate the random effects, $b(q)$, so that the dynamics is kept away from zero. Note what matters is the ratio of the deterministic drift to the size of variance. Even when $a(q)$ is positive in the neighborhood of zero, $q(t)$ may converge to zero with a positive probability if $b(q)^{2}$ is much larger than $a(q)$. Because of the large variance, the optimists may suffer from a run of sizable bad luck, which makes their wealth a negligible proportion of the total wealth, and once in that situation it is difficult for their wealth to become nonnegligible as a proportion of total wealth.

Similarly, the function $I_{2}$ measures the difficulty for $q(t)$ to converge to one. A large $I_{2}$ means that $a(q) / b(q)^{2}$ is negative when $q$ is close to one. Thus the negative deterministic drift tends to dominate the random effects, so that the dynamics is kept away from one.

Convergence of $q$ to zero (or one) does not imply convergence of individuals' wealth to zero. Individuals' wealth does not converge to zero with positive probability in finite time, and might even go to infinity if the discount factor is not very large, even if the proportion of wealth of agents of its type goes to zero. ${ }^{5}$

For our purposes, defining a function $D(q)=2 a(q) q(1-q) / b(q)^{2}$ makes the discussion easier. Note that $D(q)$ is a bounded, continuously differentiable function in the interval $[0,1]$. Note also that both the $a($.$) and the b($.$) functions$ have a factor of $q(1-q)$ which cancels out in the expression for $D($.$) , so that$ $D($.$) is well-defined and need not be zero when q$ is zero or one.

Depending on the values of $D(q)$ at $q=0$ and $q=1$, there are four possibilities for the asymptotic dynamics of $q$, which are identified in the following proposition.

Proposition 2. a) If $D(0) \geq 1$ and $D(1) \leq-1$, the process converges to neither zero nor one; if in addition $D(0)>1$ and $D(1)<-1$, there is a unique ergodic distribution. b) If $D(0) \geq 1$ and $D(1)>-1$, the process converges to one almost surely. c) If $D(0)<1$ and $D(1) \leq-1$, the process converges to zero almost surely. d) If $D(0)<1$ and $D(1)>-1$, the process converges to one with probability $I_{1} /\left(I_{1}+I_{2}\right)$ and to zero with complementary probability.

Proof. See the Appendix.

The stochastic process for $q(t)$ has two absorbing states, zero and one, because there is no deterministic drift and no stochastic variation at these states. This is why the values of $D(0)$ and $D(1)$ are so important. The function $D($.$) compares$ the strength of the deterministic drift to the variance of the noise term.

\footnotetext{
${ }^{5}$ The proof of this statement is available upon request.
} 
Table 1. Values of $D(0), D(1)$, and the asymptotic properties of the $q(t)$ process

\begin{tabular}{lll} 
& $D(1) \leq-1$ & $D(1)>-1$ \\
\hline$D(0) \geq 1$ & $\begin{array}{l}\text { (a) ergodic distribution, if both } \\
\text { inequalities in } D(\cdot) \text { are strict; otherwise the } \\
\text { distribution depends on the initial value, } \\
q(0)\end{array}$ & $\begin{array}{l}\text { (b) a.s. convergence to } 1 \text {; this is the } \\
\text { limiting case of d) when } I_{1} \text { is infinite } \\
\text { and } I_{2} \text { is finite }\end{array}$ \\
& $\begin{array}{ll}\text { (c) a.s. convergence to } 0 \text {; this is the limiting } \\
\text { case of d) when } I_{1} \text { is finite and } I_{2} \text { is infinite }\end{array}$ & $\begin{array}{l}\text { (d) convergence to } 1 \text { with probability } \\
I_{1} /\left(I_{1}+I_{2}\right), \text { and to } 0 \text { with probability } \\
I_{2} /\left(I_{1}+I_{2}\right)\end{array}$ \\
\hline
\end{tabular}

Table 1 gives a visual exposition of Proposition 2. The value of $D(0)$ is important in determining whether the process converges to zero with positive probability. If $D(0)<1$, then the process $q(t)$ converges to zero with nonzero probability. The probability becomes one when $D(1) \leq-1$ also holds. If $D(0) \geq 1$, however, the process never converges to zero. Similarly, the value of $D(1)$ is important in determining whether the process $q(t)$ converges to one with a positive probability.

We will now show that all of the cases presented in Proposition 2 are possible for some open nonempty set of the parameter values of this model. Suppose that $c^{\mathrm{o}}=c^{\mathbf{p}}=c$ and $\pi^{\mathrm{o}}=\pi^{\mathfrak{p}}=\pi$, where $c$ and $\pi$ are some constants. Since the inequalities in cases a) through d) partition the parameter space, showing that all the cases are possible for an open nonempty set of the subset of the parameter space where the above equalities hold guarantes that all cases arc also possiblc for an open nonempty set of the whole parameter space. Under the assumption that $c^{\mathrm{o}}=c^{\mathrm{p}}=c$ and $\pi^{\mathrm{o}}=\pi^{\mathrm{p}}=\pi$ :

$$
\begin{aligned}
D(0) & =\frac{2\left(\lambda^{\mathrm{p}}\left(1-\lambda^{\mathrm{p}}\right)\right)^{2}}{\left(\lambda^{\mathrm{p}}-\lambda^{\mathrm{p}}\right) \sigma^{2}}\left[\frac{\pi-\lambda^{\mathrm{p}} c}{\lambda^{\mathrm{p}}\left(1-\lambda^{\mathrm{p}}\right)}-\frac{\lambda^{\mathrm{p}} \sigma^{2}}{\left(\lambda^{\mathrm{p}}\left(1-\lambda^{\mathrm{p}}\right)\right)^{2}}\right] \\
& =\frac{2 \lambda^{\mathrm{p}}}{\lambda^{\mathrm{p}}-\lambda^{\mathrm{p}}}\left[\frac{\left(\pi-\lambda^{\mathrm{p}} c\right)\left(1-\lambda^{\mathrm{p}}\right)}{\sigma^{2}}-1\right] .
\end{aligned}
$$

Thus, $D(0) \geq 1$ if and only if

$$
2 \frac{\pi-\lambda^{p} c}{\sigma^{2}} \geq \frac{\lambda^{\mathrm{o}}+\lambda^{\mathrm{p}}}{\lambda^{\mathrm{p}}\left(1-\lambda^{\mathrm{p}}\right)}
$$


Also:

$$
\begin{aligned}
D(1) & =\frac{2\left(\lambda^{0}\left(1-\lambda^{0}\right)\right)^{2}}{\left(\lambda^{0}-\lambda^{\mathrm{p}}\right) \sigma^{2}}\left[\frac{\pi-\lambda^{0} c}{\lambda^{0}\left(1-\lambda^{0}\right)}-\frac{\lambda^{0} \sigma^{2}}{\left(\lambda^{0}\left(1-\lambda^{0}\right)\right)^{2}}\right] \\
& =\frac{2 \lambda^{0}}{\lambda^{0}-\lambda^{\mathrm{p}}}\left[\frac{\left(\pi-\lambda^{0} c\right)\left(1-\lambda^{0}\right)}{\sigma^{2}}-1\right]
\end{aligned}
$$

So $D(1) \leq-1$ if and only if

$$
2 \frac{\pi-\lambda^{o} c}{\sigma^{2}} \leq \frac{\lambda^{0}+\lambda^{p}}{\lambda^{0}\left(1-\lambda^{0}\right)}
$$

Thus, we can distinguish the four cases a) through d) in the proposition by comparing

$$
2 \frac{\pi-\lambda^{\mathrm{p}} c}{\sigma^{2}} \text { to } \frac{\lambda^{\mathrm{o}}+\lambda^{\mathrm{p}}}{\lambda^{\mathrm{p}}\left(1-\lambda^{\mathrm{p}}\right)}
$$

and

$$
2 \frac{\pi-\lambda^{0} c}{\sigma^{2}} \text { to } \frac{\lambda^{0}+\lambda^{p}}{\lambda^{0}\left(1-\lambda^{0}\right)}
$$

As an example, Fig. 1 identifies the four cases on $\lambda^{0}-\lambda^{p}$ space. It is obvious that all cases are possible for a nontrivial set of parameters.

Although the discussion above is useful in establishing that all the cases are indeed possible, the conditions derived there are hard to interpret. By rewriting the inequalities in $D(0)$ and $D(1)$ in Proposition 2, we can get a more intuitive interpretation of those conditions. For example, noting that

$$
D(0)=\frac{2\left(\lambda^{\mathrm{p}}\left(1-\lambda^{\mathrm{p}}\right)\right)^{2}}{\left(\lambda^{\mathrm{o}}-\lambda^{\mathrm{p}}\right) \sigma^{2}}\left[\frac{\pi^{\mathrm{p}}-c^{\mathrm{p}} \lambda^{\mathrm{p}}}{\lambda^{\mathrm{p}}\left(1-\lambda^{\mathrm{p}}\right)}-\frac{\lambda^{\mathrm{p}} \sigma^{2}}{\left(\lambda^{\mathrm{p}}\left(1-\lambda^{\mathrm{p}}\right)\right)^{2}}-\frac{c^{\mathrm{o}}-c^{\mathrm{p}}}{\lambda^{\mathrm{o}}-\lambda^{\mathrm{p}}}\right] .
$$

One can show that $D(0) \geq 1$ is equivalent with

$$
\left(\lambda^{\mathfrak{p}}-\lambda^{*}(0)\right)^{2}-\left(\lambda^{\mathfrak{o}}-\lambda^{*}(0)\right)^{2}-2\left(c^{\mathrm{o}}-c^{\mathfrak{p}}\right) \frac{\left(\lambda^{\mathrm{p}}\left(1-\lambda^{\mathrm{p}}\right)\right)^{2}}{\sigma^{2}} \geq 0,
$$

where

$$
\lambda^{*}(0) \equiv \frac{\lambda^{\mathrm{p}}\left(1-\lambda^{\mathrm{p}}\right)\left(\pi^{\mathrm{p}}-c^{\mathrm{p}} \lambda^{\mathrm{p}}\right)}{\sigma^{2}} .
$$




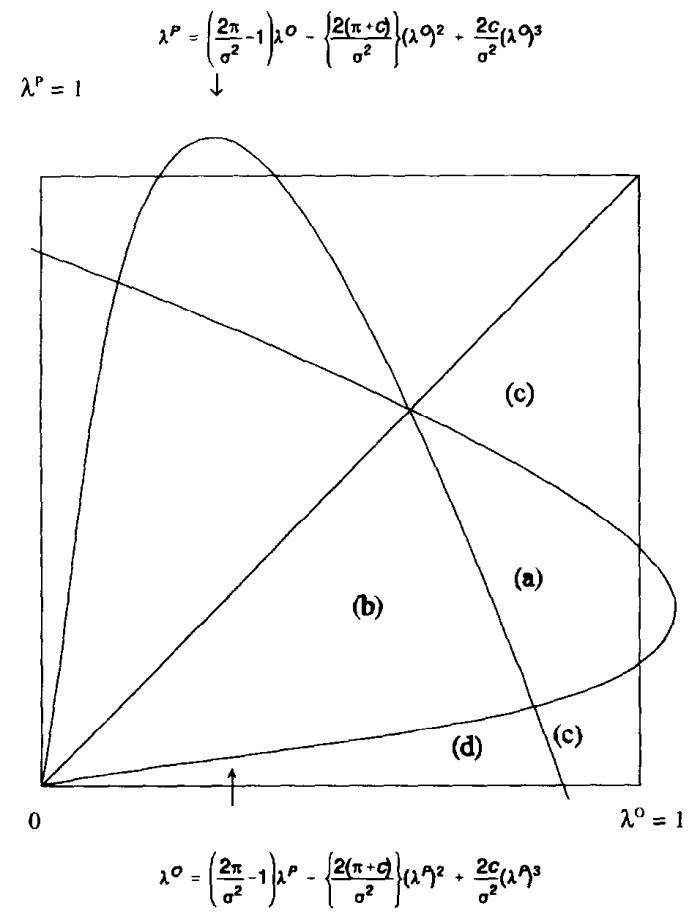

Fig. 1. The four cases in Proposition 2.
(a) $\frac{\lambda^{\circ}+\lambda^{\mathrm{p}}}{\lambda^{\mathrm{o}}\left(1-\lambda^{\mathrm{o}}\right)} \geq 2 \frac{\pi-\lambda^{\mathrm{o}} c}{\sigma^{2}}$ and $2 \frac{\pi-\lambda^{\mathrm{p}} c}{\sigma^{2}} \geq \frac{\lambda^{\mathrm{o}}+\lambda^{\mathrm{p}}}{\lambda^{\mathrm{p}}\left(1-\lambda^{\mathrm{p}}\right)}$
(b) $\frac{\lambda^{0}+\lambda^{p}}{\lambda^{0}\left(1-\lambda^{0}\right)}<2 \frac{\pi-\lambda^{0} c}{\sigma^{2}}$ and $2 \frac{\pi-\lambda^{p} c}{\sigma^{2}} \geq \frac{\lambda^{0}+\lambda^{p}}{\lambda^{p}\left(1-\lambda^{p}\right)}$
(c) $\frac{\lambda^{0}+\lambda^{p}}{\lambda^{0}\left(1-\lambda^{0}\right)} \geq 2 \frac{\pi-\lambda^{o} c}{\sigma^{2}}$ and $2 \frac{\pi-\lambda^{p} c}{\sigma^{2}}<\frac{\lambda^{o}+\lambda^{p}}{\lambda^{p}\left(1-\lambda^{p}\right)}$
(d) $\frac{\lambda^{\circ}+\lambda^{p}}{\lambda^{\circ}\left(1-\lambda^{\circ}\right)}<2 \frac{\pi-\lambda^{\circ} c}{\sigma^{2}}$ and $2 \frac{\pi-\lambda^{p} c}{\sigma^{2}}<\frac{\lambda^{\circ}+\lambda^{p}}{\lambda^{p}\left(1-\lambda^{p}\right)}$ 
Note that $\lambda^{*}(0)$ is the proportion of wealth that the agents would invest in the risky asset if they had logarithmic utility and their beliefs were correct when $q=0$ (no optimists). ${ }^{6}$ Inequality (6) is useful for interpreting the results in Proposition 2 in an intuitive way. Suppose, for the moment, that $c^{0}$ is equal to $c^{\mathfrak{p}}$. Then the last term on the left-hand side of (6) vanishes, and whether the inequality holds depends on whether the optimists' portfolio $\lambda^{\circ}$ is closer to $\lambda^{*}(0)$ than the pessimists' portfolio $\lambda^{p}$. First, let us consider the case when inequality (6) holds, that is when $D(0) \geq 1$. In this case, $\lambda^{\circ}$ is closer to $\lambda^{*}(0)$, which is the optimal strategy of a logarithmic utility maximizer when there are no optimists, implying that the optimists do better than the pessimists' when the share of the optimists' wealth is close to zero. Thus, whenever the optimists' share of wealth comes close to zero, the optimists start to do better and increase their share of the wealth. Therefore, the optimists' share of the wealth never converges to zero in this case. If $D(0)<1$, so that inequality (6) does not hold, the share of the optimists' wealth can end up being zero. When $D(0)<1$, whenever the optimists' share of wealth is close to zero, the pessimists do better and can increase their share until it converges to one. To interpret inequality (6), we have so far assumed that $c^{\mathfrak{o}}=c^{\mathfrak{p}}$. Holding everything else equal, a $c^{\mathfrak{o}}$ greater than $c^{\mathfrak{p}}$ makes it easier for the optimists' share of the wealth to converge to zero with positive probability. Since a high consumption rate slows down wealth accumulation, the result makes sense. In a similar way, we can intuitively understand why the optimists' share of the wealth never converges to one when $D(1) \leq-1$.

Our model may appear similar to the one developed by Blume and Easley (1992). Our results are, however, somewhat different from those by Blume and Easley. In their model, if two types of investors invest constant, but different, proportions of wealth in the risky asset, as they do in our model, one type of investor sees its wealth eventually become negligible relative to the other type's

${ }^{6}$ If an investor has logarithmic utility and expects the risky asset to follow a stochastic process given by $\alpha(t) \mathrm{d} t+\beta(t) \mathrm{d} B(t)$, and the riskless asset return to be $r$, then the optimal proportion of wealth invested in the risky asset is $(\alpha(t)-r) / \beta(t)^{2}$. In our model, if the investor has correct beliefs, then

$$
\begin{aligned}
& x(t)=\frac{\pi(q(t))-\lambda^{0} c^{\mathrm{o}} q(t)-\lambda^{\mathrm{p}} c^{\mathrm{p}}(1-q(t))}{\lambda^{\mathrm{o}}\left(1-\lambda^{\mathrm{o}}\right) q(t)+\lambda^{\mathrm{p}}\left(1-\lambda^{\mathrm{p}}\right)(1-q(t))}+r, \\
& \beta(t)=\frac{\sigma}{\lambda^{\mathrm{o}}\left(1-\lambda^{\mathrm{o}}\right) q(t)+\lambda^{\mathrm{p}}\left(1-\lambda^{\mathrm{p}}\right)(1-q(t))} .
\end{aligned}
$$

Thus, $(\alpha(t)-r) / \beta(t)^{2}$ is given by

$$
\frac{1}{\sigma^{2}}\left[\pi(q(t))-\lambda^{\mathrm{o}} c^{\mathrm{o}} q(t)-\lambda^{\mathrm{p}} c^{\mathrm{p}}(1-q(t))\right]\left[\lambda^{\mathrm{o}}\left(1-\lambda^{\mathrm{o}}\right) q(t)+\lambda^{\mathrm{p}}\left(1-\lambda^{\mathrm{p}}\right)(1-q(t))\right] .
$$

When $q=0$, the above expression simplifies to

$$
\frac{1}{\sigma^{2}} \lambda^{p}\left(1-\lambda^{p}\right)\left(\pi^{p}-\lambda^{p} c^{p}\right)
$$


wealth (Blume and Easley, 1992, Propositions 3.1 and 3.2, p. 16). In our model, one type of investor may eventually dominate the other [cases (b) and (c) in Proposition 2], but two types may co-exist even in the long run [case (a)], or both types have a chance of dominating the market and the probability of one type eliminating the other depends on the initial condition [case (d)].

What is responsible for the difference is the presence of changes in the price level in the asset returns in our model. The Blume and Easley model considers assets that pay state contingent payoffs, which do not depend on changes in the prices. In our model the payouts of the risky asset include increases in the price. Since the price changes as the distribution of wealth between the two types of investors changes, the true process of the rate of return also changes in our model.

If we allow investors in our model to have nonconstant portfolio strategy, we can prove a proposition that is similar to Theorem 5.2 in Blume and Easley (1992). If one type of investor chooses a portfolio strategy that always differs more from the (nonconstant) optimal strategy that would be chosen by an investor with correct beliefs and logarithmic utility than the other strategy does, then the wealth share of such investor becomes asymptotically negligible.

Proposition 3. Suppose we have a riskless asset with rate of return $r$ and a risky asset with rate of return

$$
\frac{\mathrm{d} P(t)+\mathrm{d} D(t)}{P(t)}=\alpha(t) \mathrm{d} t+\beta(t) \mathrm{d} B(t),
$$

where $\alpha(t), \beta(t)$ are bounded, measurable functions for all $t$ and $\bar{\beta}>|\beta(t)|>\beta>0$ for all t. Let $\lambda_{1}(t), \lambda_{2}(t)$ be the investment shares of agents $I$ and 2 in the risky asset, which are also assumed to be bounded and measurable, and suppose that both agents' consumption rates are $c^{1}(t)$ and $c^{2}(t)$. We will denote $k_{i}(t)=\lambda_{i}(t)-$ $(\alpha(t)-r) / \beta^{2}(t)$ for $i=1$, 2. If $\left|k_{1}(t)\right|-\left|k_{2}(t)\right|>k^{*}>0$ and $c_{1}(t)-c_{2}(t)>c^{*}>0$ almost surely, $W^{1}(t) / W^{2}(t)$ converges to zero almost surely.

Proof. See the Appendix.

Note that $k_{i}(t)$ is a measure of distance between $\lambda_{i}(t)$ and $(\alpha(t)-r) / \beta(t)^{2}$, which is the portfolio strategy that would be followed by an investor with logarithmic utility and the correct beliefs. The parameters in the process of asset returns, $\alpha(t)$ and $\beta(t)$, are functions of $t$, and $(\alpha(t)-r) / \beta^{2}(t)$ also changes over time. The assumption of time-varying $\alpha$ and $\beta$ reflects the results in our model [Proposition 2(a)].

Comparing Proposition 3 to Theorem 5.2 in Blume and Easley (1992) clarifies an important difference between our model and theirs. In their model, 'fitness' 
of a strategy can be measured by the distance between the strategy and the one that would be used by an investor with logarithmic utility and correct beliefs, which turns out to be a constant portfolio strategy. In our model, fitness of a strategy can be measured also by its distance from the optimal strategy of a logarithmic utility maximizer with correct beliefs. But the fittest strategy is not a constant portfolio strategy. Thus, it is possible for the optimists' strategy to be closer to the fittest strategy when the optimists' share of wealth is small, and the pessimist strategy becomes fitter when the pessimists' share of wealth is small, allowing two strategies to coexist even in the long run.

\section{Asset returns process and $\mathrm{ARCH}$}

Our model implies that the process followed by asset returns has a timevarying variance. As Eq. (5) suggests, the standard deviation of the returns depends on $q(t)$, which fluctuates over time.

Time-varying conditional heteroskedasticity of asset returns has been documented by many empirical studies that applied ARCH models to financial data. Those empirical studies found that the conditional variance of asset return is well captured by a stochastic process that depends on its own past.

A class of ARCH models called ARCH-M (ARCH in mean) model considers the dependence of the mean rate of return on the conditional variance. Thus, the mean as well as the variance of rate of return becomes time-varying in ARCH-M model. Our model also predicts the time-varying average returns.

We examine the stochastic process of the conditional variance of the asset returns implied by our model and argue that our model can give a theoretical justification for an ARCH-like model in finance.

Given what we found in Section 2 the returns process can be written as

$$
\frac{\mathrm{d} P(t)+\mathrm{d} D(t)}{P(t)}=\alpha(q(t)) \mathrm{d} t+V(q(t)) \mathrm{d} B(t)
$$

where

$$
\begin{aligned}
& \alpha(q)=\frac{\pi(q)-\lambda^{0} c^{\circ} q-\lambda^{p} c^{p}(1-q)}{\lambda^{0}\left(1-\lambda^{o}\right) q+\lambda^{p}\left(1-\lambda^{p}\right)(1-q)}+r \\
& V(q)=\frac{\sigma}{\lambda^{0}\left(1-\lambda^{0}\right) q+i^{\mathcal{P}}\left(1-\lambda^{\mathcal{P}}\right)(1-q)} .
\end{aligned}
$$


Since $V$ is a monotonic function of $q$ we can write $q$ as a function of $V$,

$$
q=\frac{1}{\lambda^{\mathrm{o}}\left(1-\lambda^{\mathrm{O}}\right)-\lambda^{\mathrm{P}}\left(1-\lambda^{\mathrm{P}}\right)}\left(\frac{\sigma}{V}-\lambda^{\mathrm{P}}\left(1-\lambda^{\mathrm{P}}\right)\right) \equiv g(V) .
$$

Applying Itô's Lemma to the definition of $V$, and noting that $\mathrm{d} q(t)=$ $a(q(t)) \mathrm{d} t+b(q(t)) \mathrm{d} B(t)$ and $q=g(V)$,

$$
\begin{aligned}
\mathrm{d} V(t)= & V^{\prime}(g(V(t))) \alpha(g(V(t))) \mathrm{d} t+\frac{1}{2} V^{\prime \prime}(g(V(t))) V^{2}(t) \mathrm{d} t \\
& +V^{\prime}(g(V(t))) V(t) \mathrm{d} B(t),
\end{aligned}
$$

which is a nonlinear, continuous time version of $\mathrm{ARCH}$, since the conditional variance of the asset returns is time-varying (thus heteroskedastic) and depends on its past (thus autoregressive).

The relationship goes further since the conditional mean of the returns, $\alpha(q(t))$, also depends on $q(t)$, and therefore on the variance since $q=g(V)$. So we can write

$$
\frac{\mathrm{d} P(t)+\mathrm{d} D(t)}{P(t)}=\alpha(g(V(t))) \mathrm{d} t+V(t) \mathrm{d} B(t) .
$$

This can be considered as a nonlinear version of ARCH-M. ${ }^{\text {? }}$

\section{Volume of trade and volatility}

Another set of empirical implications of interest is those that relate the volume of trade and volatility. Many researchers have looked at the relation between trading volume and volatility and often found a positive relation (see Gallant, Rossi, and Tauchen, 1992, for example). We show that our model has an empirical implication for this relation. Under some parameter values, our model implies a positive relation between volatility and trading volume. We consider two alternative definitions of the trading volume: the total value of the shares that change hands and the total number of shares that change hands. The first

\footnotetext{
${ }^{7}$ Another type of statistical model that can be given a behavioral foundation by our model is the STAR model (see Teräsvirta and Anderson, 1991; Anderson, 1992). A STAR model assumes that the stochastic process followed by a certain variable is a weighted average of two distinct AR processes and the weight depends on lagged values of the variable under consideration. It can be easily shown that the process for returns in our model follows a stochastic process which resembles closely a (continuous-time) STAR model.
} 
definition gives us a result that is easier to interpret. In order to simplify the mathematical expressions, we introduce the following notation:

$$
\begin{aligned}
& A(t)=\lambda^{\mathrm{o}}\left(1-\lambda^{\mathrm{o}}\right) q(t)+\lambda^{\mathrm{p}}\left(1-\lambda^{\mathrm{p}}\right)(1-q(t)), \\
& A^{\prime}(t)=\lambda^{\mathrm{o}} q(t)+\lambda^{\mathrm{p}}(1-q(t)), \\
& \pi_{c}(t)=\pi(q(t))-\lambda^{\mathrm{o}} c^{\mathrm{o}} q(t)-\lambda^{\mathrm{p}} c^{\mathrm{p}}(1-q(t)) .
\end{aligned}
$$

$N^{\circ}(t)$ is the number of shares held by the optimists. By definition of $\lambda^{\circ}$, $N^{\circ}(t)=\lambda^{\circ} W^{\circ}(t) / P(t)$.

First consider the total value of the shares that change hands. Since shares are traded unidirectionally, between optimists and pessimists, instantaneous transaction volume is just $\mathrm{d}\left(N^{\mathrm{o}}(t) P(t)\right)=\lambda^{\circ} \mathrm{d} W^{\mathrm{o}}(t)$. Substituting Eq. (5) into Eq. (1) we get

$$
\frac{\mathrm{d} W^{\circ}(t)}{W^{\circ}(t)}=\left(\lambda^{\circ} \frac{\pi_{c}(t)}{A(t)}+r-c^{\circ}\right) \mathrm{d} t+\lambda^{\circ} \frac{\sigma}{A(t)} \mathrm{d} B(t) .
$$

Since $\sigma / A(t)$ is asset return volatility we have the desired relation. If we abstract from the fact that $\pi_{c}(t)$ depends on $q(t)$, which is a monotonic function of volatility, we can find a linear relationship between volatility and volume.

If we define the transaction volume as the number of shares that change hands, calculations become more involved. Using Itô's formula:

$$
\frac{\mathrm{d} N^{\mathrm{o}}(t)}{N^{\mathrm{o}}(t)}=\frac{\mathrm{d} W^{\mathrm{o}}(t)}{W^{\mathrm{o}}(t)}-\frac{\mathrm{d} P(t)}{P(t)}-\frac{\mathrm{d} W^{\mathrm{o}}(t) \mathrm{d} P(t)}{W^{\mathrm{o}}(t) P(t)}+\frac{(\mathrm{d} P(t))^{2}}{P(t)^{2}} .
$$

Assume that the firm does not buy back shares with the profits (and only pays dividends) so that trading is done exclusively by investors. Then

$$
\frac{1}{N(t)} \frac{\mathrm{d} \pi^{*}(t)}{P(t)}=\frac{\mathrm{d} D(t)}{P(t)}=(\pi(q(t)) \mathrm{d} t+\sigma \mathrm{d} B(t)) \frac{W(t)}{P(t) N(t)}=\frac{\pi(q(t)) \mathrm{d} t+\sigma \mathrm{d} B(t)}{A^{\prime}(t)},(7)
$$

where the last equality follows from the market clearing condition, Eq. (3). Using Eqs. (7) and (5),

$$
\begin{aligned}
\frac{\mathrm{d} P(t)}{P(t)}= & \frac{\mathrm{d} P(t)+\mathrm{d} D(t)}{P(t)}-\frac{\mathrm{d} D(t)}{P(t)} \\
& =\left(\frac{\pi_{\mathrm{c}}(t)}{A(t)}+r-\frac{\pi(q(t))}{A^{\prime}(t)}\right) \mathrm{d} t+\left(\frac{\sigma}{A(t)}-\frac{\sigma}{A^{\prime}(t)}\right) \mathrm{d} B(t) .
\end{aligned}
$$


Thus,

$$
\begin{aligned}
\frac{\mathrm{d} N^{\mathrm{o}}(t)}{N^{\mathrm{o}}(t)}= & \left(\lambda^{\mathrm{o}} \frac{\pi_{\mathrm{c}}(t)}{A(t)}+\lambda^{\mathrm{o} r}+\left(1-\lambda^{\mathrm{o}}\right) r-\left(\frac{\pi_{c}(t)}{A(t)}+r-\frac{\pi(q(t))}{A^{\prime}(t)}\right)\right. \\
& \left.-\lambda^{\mathrm{o}} \frac{\sigma}{A(t)}\left(\frac{\sigma}{A(t)}-\frac{\sigma}{A^{\prime}(t)}\right)+\left(\frac{\sigma}{A(t)}-\frac{\sigma}{A^{\prime}(t)}\right)^{2}\right) \mathrm{d} t \\
& +\left(\lambda^{\mathrm{o}} \frac{\sigma}{A(t)}-\left(\frac{\sigma}{A(t)}-\frac{\sigma}{A^{\prime}(t)}\right)\right) \mathrm{d} B(t), \\
= & \left(\lambda^{\mathrm{o}} \frac{\pi_{c}(t)}{A(t)}-\left(\frac{\pi_{c}(t)}{A(t)}-\frac{\pi(q(t))}{A^{\prime}(t)}\right)\right. \\
& \left.-\lambda^{\mathrm{o}} \frac{\sigma}{A(t)}\left(\frac{\sigma}{A(t)}-\frac{\sigma}{A^{\prime}(t)}\right)+\left(\frac{\sigma}{A(t)}-\frac{\sigma}{A^{\prime}(t)}\right)^{2}\right) \mathrm{d} t \\
& +\left(\lambda^{\circ} \frac{\sigma}{A(t)}-\left(\frac{\sigma}{A(t)}-\frac{\sigma}{A^{\prime}(t)}\right)\right) \mathrm{d} B(t) .
\end{aligned}
$$

Since $q(t)$ is a monotonic function of volatility this last expression relates volume to volatility.

To get a better intuition, assume $\pi_{c}(t)=\pi(q(t))$ (i.e., $\left.c^{\mathrm{o}}=c^{\mathrm{p}}=0\right)$. Noting that $\left(\lambda^{\mathrm{o}}-1\right) A^{\prime}(t)+A(t)=\left(\lambda^{\mathrm{o}}-\lambda^{\mathrm{p}}\right) \lambda^{\mathrm{p}}(1-q(t))$ and letting $A^{\prime \prime}(t)=\left(\lambda^{\mathrm{o}}-\lambda^{\mathrm{p}}\right) \lambda^{\mathrm{p}}(1-$ $q(t))$, we have

$$
\begin{aligned}
& \frac{\mathrm{d} N^{\mathrm{o}}(t)}{N^{\mathrm{o}}(t)}=\left(\pi(q(t)) \frac{A^{\prime \prime}(t)}{A(t) A^{\prime}(t)}-\sigma^{2}\left(\frac{\lambda^{\mathrm{o} 2}+\lambda^{p^{2}}(1-q(t))}{A(t) A^{\prime}(t)}\right) \frac{A^{\prime \prime}(t)}{A(t) A^{\prime}(t)}\right) \mathrm{d} t \\
& +\frac{A^{\prime \prime}(t)}{A(t) A^{\prime}(t)} \mathrm{d} B(t) .
\end{aligned}
$$

Note that $A^{\prime \prime}(t) / A^{\prime}(t)=\left(\lambda^{\mathrm{o}}-\lambda^{\mathrm{p}}\right) \lambda^{\mathrm{p}}(1-q(t)) /\left[\lambda^{\mathrm{o}}-\left(\lambda^{\mathrm{o}}-\lambda^{\mathrm{p}}\right)(1-q(t))\right]$ is decreasing in $q(t)$. We can show that both the conditional mean of volume and its variance are decreasing functions of $q(t)$ if $\lambda^{\mathrm{p}}\left(1-\lambda^{\mathrm{p}}\right) \leq \lambda^{\mathrm{o}}\left(1-\lambda^{\mathrm{o}}\right), \pi(q(t)) / \sigma$ is large and $\pi^{\mathfrak{p}} \geq \pi^{\circ}$. ${ }^{8}$ Thus, under these conditions, since asset price volatility is

\footnotetext{
${ }^{8}$ If $\pi(q(t)) / \sigma$ is large, then to determine whether the conditional mean of volume is a decreasing function of $q(t)$ we only need to know whether $\pi(q(t))\left[A^{\prime}(t) / A(t) A^{\prime \prime}(t)\right]$ is decreasing in $q(t)$. If $\pi^{\circ}<\pi^{\mathrm{p}}, \pi(q(t))$ is decreasing in $q(t)$ and if $\lambda^{\mathrm{p}}\left(1-\lambda^{\mathrm{p}}\right)<\lambda^{\mathrm{o}}\left(1-\lambda^{\circ}\right), 1 / A(t)$ is decreasing in $q(t)$. We already showed that $A^{\prime \prime}(t) / A^{\prime}(t)$ is decreasing in $q(t)$. Thus, if $\pi(q(t)) / \sigma$ is large, $\pi^{0}<\pi^{\mathfrak{p}}$ and
} 
a decreasing function of $q(t)$, asset price volatility and volume of trade are positively correlated.

\section{Fundamentalists and contrarians}

This section studies a model in which some agents use marketwide information when making their investment decisions. The first type of agent is called fundamentalist because agents of this type believe they know the process which generates the profits of the firm, and they act in a way that would be optimal if they were the only type of agent in the market. The second type of agent is called contrarian. Agents of this type subscribe to the theory that the majority of agents are adopting wrong positions in financial markets, and they adopt the opposite position.

We consider the same economic structure and use the same notation as in Section 2. Let $W^{\mathrm{F}}(t)$ and $W^{\mathrm{C}}(t)$ be the total wealth of fundamentalists and contrarians respectively. Then the total wealth in the economy, $W(t)$, is given by $W^{\mathrm{F}}(t)+W^{\mathrm{C}}(t)$. Let $\lambda^{\mathrm{F}}$ and $\lambda^{\mathrm{C}}(t)$ be the shares of the risky asset in the portfolios of fundamentalists and contrarians respectively. Note that contrarians follow a time-varying portfolio strategy. Contrarians assume that the market is wrong, and put $1-P(t) N(t) / W(t)$ into the risky asset, because the market portfolio puts $P(t) N(t) / W(t)$ into the risky asset. Thus, $\lambda^{\mathrm{C}}(t)=1-P(t) N(t) / W(t)$.

Let $q(t) \equiv W^{\mathrm{C}}(t) /\left[W^{\mathrm{C}}(t)+W^{\mathrm{F}}(t)\right]$ denote the share of total wealth held by the contrarians. Market clearing implies

$$
\left(1-\frac{P(t) N(t)}{W(t)}\right) q(t)+\lambda^{\mathrm{F}}(1-q(t))=\frac{P(t) N(t)}{W(t)}
$$

or

$$
\left(1-\lambda^{\mathrm{F}}+\frac{2 \lambda^{\mathrm{F}}-1}{1+q(t)}\right) W(t)=P(t) N(t) .
$$

Consumption for both types of agents is assumed to be a fixed proportion of their wealth, $c^{\mathrm{F}}$ for the fundamentalists and $c^{\mathrm{C}}$ for the contrarians. Then, the

$\lambda^{p}\left(1-\lambda^{p}\right)<\lambda^{0}\left(1-\lambda^{0}\right)$, the conditional mean of volume is decreasing in $q(t)$. The conditional variance of volume is $A^{\prime \prime}(t) / A(t) A^{\prime}(t)$. If $\lambda^{\mathrm{p}}\left(1-\lambda^{\mathrm{p}}\right)<\lambda^{\mathrm{o}}\left(1-\lambda^{\mathrm{o}}\right)$, the conditional variance of volume is decreasing in $q(t)$. 
evolution of wealth for each type of investor is given by

$$
\begin{array}{r}
\mathrm{d} W^{i}(t)=\left(\lambda^{i}(t) \frac{\mathrm{d} P(t)+\mathrm{d} D(t)}{P(t)}+\left(1-\lambda^{i}(t)\right) r \mathrm{~d} t-c^{i} \mathrm{~d} t\right) W^{i}(t), \\
i=\mathrm{C}, \mathrm{F} .
\end{array}
$$

The process for instantaneous profits is again assumed to follow:

$$
\mathrm{d} \pi^{*}(t)=(\pi(q(t)) \mathrm{d} t+\sigma \mathrm{d} B(t)) W(t)
$$

which is used to buy back shares and/or pay dividends [i.e., Eq. (4) holds].

Following similar steps to those taken in Section 2 to derive Proposition 1, we can prove the following proposition, which establishes the process of equilibrium asset returns and the contrarians share of wealth.

Proposition 4. Let

$$
\frac{\mathrm{d} P(t)+\mathrm{d} D(t)}{P(t)}=a(t) \mathrm{d} t+b(t) \mathrm{d} B(t)
$$

and

$$
\mathrm{d} q(t)=c(t)+g(t) \mathrm{d} B(t) .
$$

Then,

$$
\begin{aligned}
g(t)= & -\frac{q(t)(1-q(t)) \frac{2 \lambda^{\mathrm{F}}-1}{1+q(t)}}{\left(1-\lambda^{\mathrm{F}}\right) \lambda^{\mathrm{F}}+\left(2 \lambda^{\mathrm{F}}-1\right)^{2} \frac{2 q(t)^{2}}{(1+q(t))^{3}}} \sigma, \\
b(t)= & \frac{\sigma-g(t) \frac{2 \lambda^{\mathrm{F}}-1}{(1+q(t))^{2}}}{\left(1-\lambda^{\mathrm{F}}\right) \lambda^{\mathrm{F}}+\lambda^{\mathrm{F}}+\left(2 \lambda^{\mathrm{F}}-1\right)^{2} \frac{q(t)}{(1+q(t))^{2}}} \\
c(t)= & \frac{g(t)}{\sigma}\left[\pi(q(t))+\frac{2 \lambda^{\mathrm{F}}-1}{(1+q(t))^{3}} g(t)^{2}\right. \\
& +\left(1-\lambda^{\mathrm{F}}+\frac{2 \lambda^{\mathrm{F}}-1}{1+q(t)}\right)\left(-q(t) c^{\mathrm{C}}-(1-q(t)) c^{\mathrm{F}}\right)
\end{aligned}
$$




$$
\begin{gathered}
\left.-\frac{\left(2 \lambda^{\mathrm{F}}-1\right) g(t) b(t)}{(1+q(t))^{2}}\left(1-\lambda^{\mathrm{F}}+\frac{2 \lambda^{\mathrm{F}}-1}{1+q(t)}\right)\right] \\
-q(t)(1-q(t))\left(c^{\mathrm{C}}-c^{\mathrm{F}}\right) \frac{\left(1-\lambda^{\mathrm{F}}\right) \lambda^{\mathrm{F}}+\frac{\left(2 \lambda^{\mathrm{F}}-1\right)^{2} q(t)}{(1+q(t))^{2}}}{\left(1-\lambda^{\mathrm{F}}\right) \lambda^{\mathrm{F}}+\frac{\left(2 \lambda^{\mathrm{F}}-1\right)^{2} 2 q(t)^{2}}{(1+q(t))^{3}}} \\
+q(t)(1-q(t))\left(\frac{2 \lambda^{\mathrm{F}}-1}{1+q(t)}\right)\left(1-\lambda^{\mathrm{F}}+\frac{2 \lambda^{\mathrm{F}}-1}{1+q(t)}\right) b(t)^{2}, \\
a(t)=\frac{\pi(q(t))+\frac{2 \lambda^{\mathrm{F}}-1}{(1+q(t))^{3}} g(t)^{2}}{\left(1-\lambda^{\mathrm{F}}\right) \lambda^{\mathrm{F}}+\frac{\left(2 \lambda^{\mathrm{F}}-1\right) q(t)}{(1+q(t))^{2}}-\frac{2 \lambda^{\mathrm{F}}-1}{(1+q(t))^{2}} g(t) b(t)} \\
\lambda^{\mathrm{F}}-\frac{2 \lambda^{\mathrm{F}}-1}{1+q(t)} \\
-\frac{2 \lambda^{\mathrm{F}}-1}{(1+q(t))^{2}} \\
\left(1-\lambda^{\mathrm{F}}\right) \lambda^{\mathrm{F}}+\frac{\left(2 \lambda^{\mathrm{F}}-1\right) q(t)}{(1+q(t))^{2}} c(t)-\frac{c^{\mathrm{C}} q(t)+c^{\mathrm{F}}(1-q(t)}{\lambda^{\mathrm{F}}-\frac{2 \lambda^{\mathrm{F}}-1}{1+q(t)}}+r .
\end{gathered}
$$

Proof. See the Appendix.

The expressions for $c(t)$ and $g(t)$ show that the drift and the diffusion terms for the stochastic process $q(t)$ are functions of $q(t)$ itself, so we can write

$$
\mathrm{d} q(t)=a_{1}(q(t)) \mathrm{d} t+b_{1}(q(t)) \mathrm{d} B(t) .
$$

The limiting properties of the process $q(t)$ can be found again by analyzing the function $D(q)=a_{1}(q) q(1-q) / b_{1}(q)^{2}$. Since the function $D(q)$ is again bounded and continuously differentiable in $[0,1]$, Proposition 2 directly applies. It can be shown that all four cases in the proposition are possible. Similarly to the model with optimists and pessimists, the different cases hold depending on the proximity of the investment policy of fundamentalists and contrarians to the optimal policy of an investor with logarithmic utility and rational expectations. ${ }^{9}$

\footnotetext{
${ }^{9}$ There is a version of this paper, with a full discussion of these issues, as well as complete proofs of the propositions, which is available upon request.
} 


\section{Concluding remarks}

Models with heterogeneous beliefs are potentially useful in explaining anomalies in the asset markets. With a few exceptions, however, the past literature on the asset markets with heterogeneous investors did not pay much attention to dynamic aspects of the models. This paper is an attempt to advance the research on the dynamic properties of the models of asset markets with heterogeneous investors, originated by De Long, Shleifer, Summers, and Waldmann (1991) and Blume and Easley (1992) among others.

In this paper, we showed that the introduction of simple heterogeneity of beliefs into a simple model of asset pricing produces a rich dynamic of asset returns. Depending on the parameter valucs of the model, onc type of investor may eventually have an infinitely larger wealth than the other investors, but it is also possible that two types of investors co-exist even in the long run while they keep influencing the asset returns.

One important empirical implication from our analysis is the presence of smooth regime shifts in the dynamics of asset returns, which are caused by the changes in the distribution of wealth among heterogeneous investors. As we have shown in Section 4, this implication is roughly consistent with a wellknown empirical regularity in financial markets: we often find ARCH in asset returns. The model also has an implication on the relation between asset return volatility and trading volume. We have not, however, put these implications through econometric tests, which is obviously an important agenda for future work.

\section{Appendix}

\section{A.1. Model with risk averters and risk lovers}

Suppose that we have two types of agents: one that is infinitely risk-averse and the other is risk-loving. There are a risky asset and a riskless asset, and the risky asset is assumed to have a higher expected return. Hence risk lovers only invest in the risky asset and the risk averters only invest in the riskless asset. There are no heterogeneous beliefs in this model. Both agents know that the risky asset is risky and has a positive mean excess return. We use $\mathrm{L}$ to denote risk lovers and A for risk averters. Let $q$ denote the proportion of total wealth held by the risk lovers. We also assume that some proportion of the returns, $\tau$, is kept by the government. Then,

$$
\frac{\mathrm{d} W^{\mathrm{L}}(t)}{W^{\mathrm{L}}(t)}=(1-\tau) \frac{\mathrm{d} D(t)+\mathrm{d} P(t)}{P(t)}, \quad \frac{\mathrm{d} W^{\mathrm{A}}(t)}{W^{\mathrm{A}}(t)}=(1-\tau) r \mathrm{~d} t
$$


Profits are assumed to follow the stochastic process:

$$
\mathrm{d} \pi^{*}(t)=(\pi(q(t)) \mathrm{d} t+\sigma(q(t)) \mathrm{d} B(t)) W(t) .
$$

Assuming that all profits are given as dividends and noting that only $L$ agents demand the risky asset:

$$
\frac{\mathrm{d} P(t)}{P(t)}=\frac{\mathrm{d} W^{\mathrm{L}}(t)}{W^{\mathrm{L}}(t)}=(1-\tau) \frac{\mathrm{d} W^{\mathrm{L}}(t)+(\pi(q(t)) \mathrm{d} t+\sigma(q(t)) \mathrm{d} B(t)) W(t)}{W^{\mathrm{L}}(t)} .
$$

Thus,

$$
\frac{\mathrm{d} W^{\mathrm{L}}(t)}{W^{\mathrm{L}}(t)}=\frac{\pi(q(t))}{\tau q(t)} \mathrm{d} t+\frac{\sigma(q(t))}{\tau q(t)} \mathrm{d} B(t) .
$$

Noting that risk averters' wealth grows at a constant rate $(1-\tau) r$ and using Itô's formula:

$$
\frac{\mathrm{d} q(t)}{q(t)}=\left(\frac{\pi(q(t))}{\tau q(t)}-q(t) r-\frac{\sigma^{2}(q(t))}{\tau^{2} q(t)}\right) \mathrm{d} t+\frac{\sigma(q(t))}{\tau q(t)} \mathrm{d} B(t) .
$$

We necd to assume that $\sigma(q(t))=0$, when $q(t)-0$.

One can show that this model has similar empirical implications as the ones obtained for the model with optimists and pessimists. The model also implies that under some parameter values, both types of agents continue to coexist in the market forever.

\section{A.2. Proofs of propositions}

Some of the proofs are only sketched. Full proofs can be obtained from the authors upon request.

Sketch of Proof of Proposition I

Eq. (2) implies

$$
\lambda^{0} \mathrm{~d} W^{\mathrm{o}}(t)+\lambda^{\mathrm{p}} \mathrm{d} W^{\mathrm{p}}(t)=\mathrm{d} N(t)(P(t)+\mathrm{d} P(t))+N(t) \mathrm{d} P(t) .
$$

Dividing Eq. (A.1) by $W(t)$ and substituting (1), (3), and (4) into (A.1) and then collecting the terms that are multiplied by $[\mathrm{d} P(t)+\mathrm{d} D(t)] / P(t)$, we get Eq. (5) [that is, part a) of the proposition]. 
Applying Itô's formula to the definition of $q(t)$ and simplifying the resulting expression, and then substituting Eq. (5) into the resulting expression we get part b) of the proposition.

\section{Proof of Proposition 2}

The proof requires establishing two lemmas.

Lemma 1 . a) If $D(0) \geq 1, I_{1}$ is infinite. b) If $D(0)<1, I_{1}$ is finite. c) If $D(1) \leq-1, I_{2}$ is infinite. d) If $D(1)>-1, I_{2}$ is finite.

Sketch of Proof for Lemma 1

a) and b) For $q_{\delta} \in(0,1), I_{1}$ can be written

$$
\begin{aligned}
I_{1}= & \exp \left(-\int_{z}^{q_{b}} \frac{D(q)}{q(1-q)} \mathrm{d} q\right)\left\{\int_{x}^{q_{b}} \exp \left[\int_{z}^{q_{b}} \frac{D(q)}{q(1-q)} \mathrm{d} q\right] \mathrm{d} x\right\} \\
& +\int_{q_{b}}^{q(0)} \exp \left[-\int_{z}^{x} \frac{D(q)}{q(1-q)} \mathrm{d} q\right] \mathrm{d} x,
\end{aligned}
$$

but for $q_{\delta}$ sufficiently close to zero.

$$
\int_{0}^{q_{\delta}} \exp \left[\int_{x}^{q_{\delta}} \frac{D(q)}{q(1-q)} \mathrm{d} q\right] \mathrm{d} x \cong \int_{0}^{q_{\delta}} \exp \left[\int_{x}^{q_{\delta}} \frac{D(0)}{q} \mathrm{~d} q\right] \mathrm{d} x=\int_{0}^{q_{\delta}}\left(\frac{q_{\delta}}{x}\right)^{D(0)} \mathrm{d} x,
$$

which is infinite if $D(0) \geq 1$ and finite if $D(0)<1$. Since $D(x)$ is bounded,

$$
\int_{q_{s}}^{q(0)} \exp \left[-\int_{z}^{x} \frac{D(q)}{q(1-q)} \mathrm{d} q\right] \mathrm{d} x \text { and } \exp \left[-\int_{z}^{q_{s}} \frac{D(q)}{q(1-q)} \mathrm{d} q\right]
$$

are strictly positive and bounded. Thus, $I_{1}$ is infinite if $D(0) \geq 1$ and finite if $D(0)<1$.

One can show c) and d) in a similar fashion.

Lemma 2. If $D(0)>1$ and $D(1)<-1, \int_{0}^{1} M(x) \mathrm{d} x$ is finite. 
For $1>q_{\delta_{2}}>q_{\delta_{1}}>0$, one can write:

$$
\begin{aligned}
\int_{0}^{1} M(x) \mathrm{d} x= & \exp \left[\int_{z}^{q_{\delta_{1}}} \frac{D(q)}{q(1-q)} \mathrm{d} q\right]\left\{\int_{0}^{q_{\delta_{1}}} \frac{2}{b(x)^{2}} \exp \left[-\int_{x}^{q_{\delta_{1}}} \frac{D(q)}{q(1-q)} \mathrm{d} q\right] \mathrm{d} x\right\} \\
& +\int_{q_{\delta_{1}}}^{q_{\delta_{2}}} \frac{2}{b(x)^{2}} \exp \left[\int_{z}^{x} \frac{D(q)}{q(1-q)} \mathrm{d} q\right] \mathrm{d} x+\exp \left[\int_{z}^{q_{\delta_{2}}} \frac{D(q)}{q(1-q)} \mathrm{d} q\right] \\
& \times\left\{\int_{q_{\delta_{2}}}^{1} \frac{2}{b(x)^{2}} \exp \left[-\int_{x}^{q_{\delta_{2}}} \frac{D(q)}{q(1-q)} \mathrm{d} q\right] \mathrm{d} x\right\} .
\end{aligned}
$$

But for $q_{\delta_{1}}$ sufficiently close to 0 ,

$$
\begin{aligned}
& \int_{0}^{q_{\delta_{1}}} \frac{2}{b(x)^{2}} \exp \left[-\int_{x}^{q_{\delta_{1}}} \frac{D(q)}{q(1-q)} \mathrm{d} q\right] \mathrm{d} x \\
& \quad \simeq \int_{0}^{q_{\delta_{1}}} \frac{2}{b(x)^{2}} \exp \left[-D(0) \ln \left(\frac{q_{\delta_{1}}}{x}\right)\right] \mathrm{d} x<K_{1} \int_{0}^{q_{\delta_{1}}} \frac{x^{D(0)}}{x^{2}} \mathrm{~d} x,
\end{aligned}
$$

where $K_{1}$ is a positive bounded constant. This is finite if $D(0)-2>-1$.

When $q_{\delta_{2}}$ is sufficiently close to 1 ,

$$
\begin{aligned}
& \int_{q_{\delta_{2}}}^{1} \frac{2}{b(x)^{2}} \exp \left[\int_{q_{\delta_{2}}}^{x} \frac{D(q)}{q(1-q)} \mathrm{d} q\right] \mathrm{d} x \\
& \quad \simeq \int_{q_{\delta_{2}}}^{1} \frac{2}{b(x)^{2}} \exp \left[-D(1) \ln \left(\frac{1-x}{1-q_{\delta_{2}}}\right)\right] \mathrm{d} x \leq K_{2} \int_{q_{\delta_{2}}}^{1} \frac{(1-x)^{-D(1)}}{(1-x)^{2}} \mathrm{~d} x
\end{aligned}
$$

for some bounded positive constant $K_{2}$. This is finite when $-D(1)-2>-1$. Since

$$
\exp \left[\int_{z}^{q_{\delta_{1}}} \frac{D(q)}{q(1-q)} \mathrm{d} q\right], \int_{q_{\delta_{1}}}^{q_{\delta_{2}}} \frac{2}{b(x)^{2}} \exp \left[\int_{z}^{x} \frac{D(q)}{q(1-q)} \mathrm{d} q\right], \exp \left[\int_{z}^{q_{\delta_{2}}} \frac{D(q)}{q(1-q)} \mathrm{d} q\right]
$$

are all finite, the lemma follows, because $D(x)$ is bounded and $z, q_{\delta_{1}}$, and $q_{\delta_{2}}$ are in $(0,1)$.

Lemma 1 and Lemma 2 establish the proposition. 
By Eq. (1) the evolution of wealth is given by

$$
\begin{array}{r}
\mathrm{d} W^{i}(t)=\left(\lambda_{i}(t) \frac{\mathrm{d} P(t)+\mathrm{d} D(t)}{P(t)}+\left(1-\lambda_{i}(t)\right) r \mathrm{~d} t-c^{i}(t) \mathrm{d} t\right) W^{i}(t) \\
i=1,2
\end{array}
$$

Let $h(t)=\log \left(W^{1}(t) / W^{2}(t)\right)$. By Itô's lemma and Eq. (A.2),

$$
\begin{aligned}
h(t)= & h(0)+\int_{0}^{T}\left[\frac{\beta(s)^{2}}{2}\left(\left(k_{2}(s)^{2}-k_{1}(s)^{2}\right)-\left(c^{1}(s)-c^{2}(s)\right)\right)\right] \mathrm{d} s \\
& +\int_{0}^{t}\left(\lambda_{1}(s)-\lambda_{2}(s)\right) \beta(s) \mathrm{d} B(s) .
\end{aligned}
$$

Let $\bar{\beta}>|\beta(s)|>\beta, \forall s$, and $\bar{\lambda}>\left|\lambda_{1}(s)-\lambda_{2}(s)\right|>k^{*}, \forall s$. By Gihman and Skorohod's (1972) Theorem 1.1,

$$
\begin{aligned}
& \left.\mathrm{E}\left[\sup _{2^{n} \leq t \leq 2^{n+1}}\left(\frac{\int_{0}^{t}\left(\lambda_{1}(s)-\lambda^{2}(s)\right) \beta(s) \mathrm{d} B(s)}{t}\right)\right)^{2}\right] \\
& \leq \frac{1}{\left(2^{n}\right)^{2}} 4 \int_{0}^{2^{n+1}} \mathrm{E}\left[\left(\lambda_{1}(s)-\lambda_{2}(s)\right)^{2} \beta(s)^{2}\right] \mathrm{d} s \leq \frac{8(\lambda \bar{\beta})^{2}}{2^{n}}
\end{aligned}
$$

Applying Chebyshev's inequality and the Borel-Cantelli lemma we have

$$
\lim _{t \rightarrow \infty} \frac{1}{t} \int_{0}^{t}\left(\lambda_{1}(s)-\lambda_{2}(s)\right) \beta(s) \mathrm{d} B(s) \rightarrow 0, \quad \text { a.s. }
$$

By the assumption of the proposition,

$$
\frac{1}{t} \int_{0}^{t}\left[\frac{\beta(s)^{2}}{2}\left(\left(k_{2}(s)^{2}-k_{1}(s)^{2}\right)-\left(c^{1}(s)-c^{2}(s)\right)\right)\right] \mathrm{d} s \leq-\frac{\beta^{2}}{2}\left(k^{*}\right)^{2}-c^{*}<0 .
$$


Eqs. (A.3) and (A.4) imply that almost surely

$$
\limsup _{t \rightarrow \infty}\left\{\frac{1}{t} h(t)\right\} \leq-\frac{\underline{\beta}^{2}}{2} k^{*}-c^{*} .
$$

Therefore $h(t) \rightarrow-\infty$ almost surely, which implies that $W^{1}(t) / W^{2}(t) \rightarrow 0$ almost surely.

\section{Sketch of Proof of Proposition 4}

Using Itô's rule on Eq. (8), substituting Eqs. (8) and (4) into the resulting expression, and dividing by $W(t)$ we get

$$
\begin{aligned}
& -\frac{2 \lambda^{\mathrm{F}}-1}{(1+q(t))^{2}} \mathrm{~d} q(t)+\frac{2 \lambda^{\mathrm{F}}-1}{(1+q(t))^{3}}(\mathrm{~d} q(t))^{2}+\left(1-\lambda^{\mathrm{F}}+\frac{2 \lambda^{\mathrm{F}}-1}{1+q(t)}\right) \frac{\mathrm{d} W(t)}{W(t)} \\
& -\frac{2 \lambda^{\mathrm{F}}-1}{(1+q(t))^{2}} \mathrm{~d} q(t) \frac{\mathrm{d} W(t)}{W(t)} \\
& =-\pi(q(t)) \mathrm{d} t-\sigma \mathrm{d} B(t)+\frac{\mathrm{d} P(t)+\mathrm{d} D(t)}{P(t)}\left(1-\lambda^{\mathrm{F}}+\frac{2 \lambda^{\mathrm{F}}-1}{1+q(t)}\right) .
\end{aligned}
$$

Since $\mathrm{d} W(t)=\mathrm{d} W^{\mathrm{C}}(t)+\mathrm{d} W^{\mathrm{F}}(t)$, using Eq. (9) and combining the result with Eq. (8), we obtain

$$
\begin{aligned}
\frac{\mathrm{d} W(t)}{W(t)}= & \left(\frac{\mathrm{d} P(t)+\mathrm{d} D t}{P(t)}-r \mathrm{~d} t\right)\left(1-\lambda^{\mathrm{F}}+\frac{2 \lambda^{\mathrm{F}}-1}{1+q(t)}\right) \\
& +r \mathrm{~d} t-q(t) c^{\mathrm{C}} \mathrm{d} t-(1-q(t)) c^{\mathrm{F}} \mathrm{d} t .
\end{aligned}
$$

Substituting (10), (11), and (A.6) into (A.5) and collecting the terms that multiply $[\mathrm{d} P(t)+\mathrm{d} D(t)] / P(t)$, we get the expression for $[\mathrm{d} P(t)+\mathrm{d} D(t)] / P(t)$ in terms of $q(t)$ and the coefficients in (10) and (11). Substituting the result into the expression for $\mathrm{d} q(t)$ which is obtained by applying Itô's rule to the definition of $q(t)$, and then identifying coefficients in the resulting expression with (10) and (11), the proposition follows.

\section{References}

Anderson, H.M., 1992, Nonlinearities and shifts in macroeconomic regimes, Unpublished Ph.D. dissertation (University of California, San Diego, CA). 
Black, F., 1986, Noise, Journal of Finance 41, 529-543.

Blume, L. and D. Easley, 1992, Evolution and market behavior, Journal of Economic Theory 58, 9-40.

Bollerslev, T., R.Y. Chou, and K.F. Kroner, 1992, ARCH modeling in finance: A selective review of the theory and empirical evidence, with suggestions for future research, Journal of Econometrics $52,5-59$.

De Long, J.B., A. Shleifer, I.H. Summers, and R.J. Waldmann, 1990, Noise trader risk in financial markets, Journal of Political Economy 98, 703-738.

De Long, J.B., A. Shleifer, L.H. Summers, and R.J. Waldmann, 1991, The survival of noise traders in financial markets, Journal of Business 64, 1-19.

Frankel, J.A. and K.A. Froot, 1988, Chartists, fundamentalists and the demand for dollars, Greek Economic Review, 49-100.

Frankel, J.A. and K.A. Froot, 1990a, Chartists, fundamentalists and trading in the foreign exchange market, American Economic Review (Papers and Proceedings) 80, 181-185.

Frankel, J.A. and K.A. Froot, 1990b, Exchange rate forecasting techniques, survey data, and implications for the foreign exchange market, NBER working paper no. 3470.

Fudenberg, D. and C. Harris, 1992, Evolutionary dynamics with aggregate shocks, Journal of Economic Theory $57,420-441$.

Gallant, A.R., P.E. Rossi, and G. Tauchen, 1992, Stock prices and volume, Review of Financial Studies 5, 199-142.

Gihman, I.I. and A.V. Skorohod, 1972, Stochastic differential cquations (Springer Verlag, Berlin).

Merton, R.C., 1969, Lifetime portfolio selection under uncertainty: The continuous-time case, Review of Economics and Statistics 51, 247-257.

Merton, R.C., 1971, Optimal consumption and portfolio rules in a continuous-time model, Journal of Economic Theory 3, 373-413.

Merton, R.C., 1973, An intertemporal capital asset pricing model, Econometrica 41, 867-887.

Ø̈ksendal, B., 1989, Stochastic differential equations (Springer Verlag, Berlin).

Shiller, R.J., 1984, Stock prices and social dynamics, Brookings Papers on Economic Activity, 457-498.

Teräsvirta, T. and H.M. Anderson, 1991, Modelling nonlinearities in business cycles using smooth transition autoregressive models, Discussion paper no. 91-24 (Department of Economics, University of California, San Diego, CA). 